\title{
ROTATION-ACTIVITY CONNECTIONS IN MAIN SEQUENCE BINARIES
}

\author{
0. Vilhu \\ Observatory and Astrophysics \\ Laboratory \\ Univ. of Helsinki, Finland
}

\author{
S.M. Rucinski \\ Max-Planck-Institut für \\ Astrophysik \\ Garching b. München, W. Germany
}

We have studied transition-region (NV + SiIV + CIV) and chromospheric (MgII) emission observed with IUE (Vilhu and Rucinski, 1982; Rucinski and Vilhu, 1982; Ayres et al., 1981) together with coronal (soft X-ray) emission observed with the Einstein satellite (Cruddace and Dupree, 1982; Walter, 1982) for contact ( $W$ UMa) and detached main sequence binaries. The components are main sequence stars (or near ms) and rapid rotators due to spin-orbit coupling. They can thus be expected to give information of dynamo-processes in rapid rotators, although the binary effects clearly produce extra complications in this discussion.

We note that the transition region (NV+SiIV + CIV, $10^{5} \mathrm{o}$ ) fractional fluxes ( $f / f_{\text {bl }}$ ) become "saturated", i.e. unsensitive to the period and spectral type, below $P \approx 3$ days; at the same time soft $X$-rays still are quite sensitive on both quantities, as shown in Fig. 1. Notice also a clear distinction in coronal emission between detached and contact binaries.

Separate wavelength-bands (excitation levels) have quite different rotational-period dependences so that estimates of the total radiative losses are rather difficult to do. We find that the magnetically-confined coronal-loop models are a convenient way to try to estimate these losses. In the simplest models (see e.g. Rosner et al., 1978) the loop pressure $p$, multiplied by the filling factor $\xi$, is proportional to the transition region surface flux, whereas the maximum loop-temperatures $\mathrm{T}_{\text {max }}$ can be estimated using the X-ray data (for details see Vilhu and Rucinski, 1982). The total radiation from loops (mainly in EUV) can then be computed from

$$
\mathrm{f}_{\text {loops }} \propto \xi \mathrm{p} \cdot \mathrm{T}_{\max }^{1 / 2} \text { erg } \mathrm{cm}^{-2} \mathrm{~s}^{-1}
$$

Dependence of this radiation ( $f_{\text {loops }} / f_{\text {bol }}$ ) on period is shown in Fig. 2 for stars in our sample for which both IUE- and Einstein-data are available. The dashed line shows the dependence as estimated from the Ayres et al. (1981) scaling laws for fluxes $\left(\mathrm{X} \sim(\mathrm{NV}+\mathrm{SiIV}+\mathrm{CIV})^{2}\right)$ used 
together with the Walter's (1982) X(P)-relation for G stars (see Fig. 1). Comparing the Sun and the most rapidly rotating stars ( $\mathrm{N} \sim 10^{-2} \mathrm{P}_{\text {sun }}$ ) we find $\mathrm{E}_{\text {loops }} / \mathrm{f}_{\mathrm{bpl}}$ on average to increase with decreasing period slightly more steeply than the linear $\mathrm{P}^{-1}$ - relation; this rise may not, however, be necessarily linear. Note that the short-period RS CVn-stars HR 1099 and UX Ari (1099 and UX in the figure) are not strictly main sequence stars.

The chromospheric radiative losses are difficult to model, but in the Sun $\mathrm{MgII} 2800$ resonance lines ( $h$ and $k$ ) may represent roughly $30 \%$ of the identifiable emission-line cooling of the chromosphere. If a similar picture holds also in the rapidly rotating active stars (i.e. losses equal to $\mathrm{MgII} / 30 \%+$ loops), we find that in the Sun chromospheric radiation dominates while in rapid rotators most of the radiation comes out from the loops. In this way, if the total (chromo $+\mathrm{TR}+$ coro $\equiv$ chromo +100 ps) radiation is considered, only the sun in the Fig. 2 will be considerably shifted upwards by approximately 1.0 dex. In this case the rapid rotators ER Vul and $\sigma^{2} \mathrm{CrB}$ would follow quite closely the $\mathrm{P}^{-1}$ - relation when compared with the Sun. With still decreasing the period (but then a physical contact sets in) the total radiative losses "freeze in" or even slightly diminish (see Fig. 2).

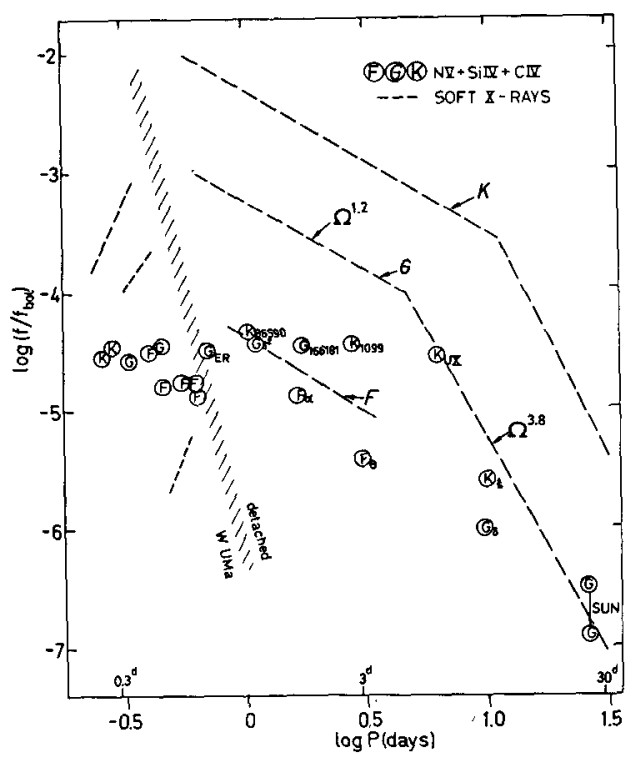

Fig. 1. Period-activity connections for transition region $(\mathrm{NV}+\mathrm{SiIV}+\mathrm{CIV})$ and coronal $(X)$ radiation.

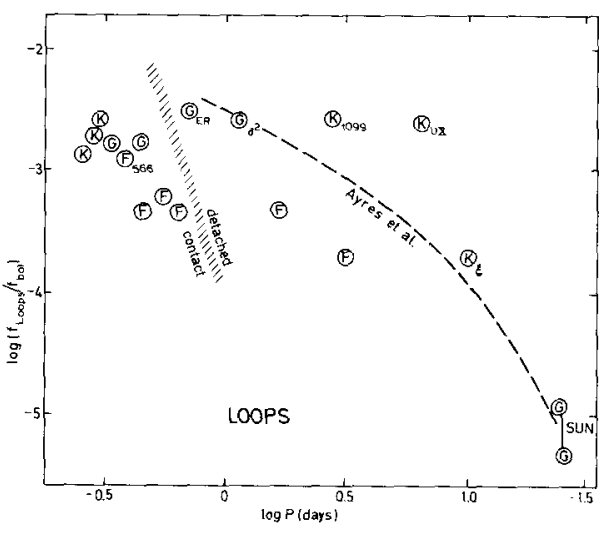

Fig. 2. Period-dependence of the total radiation from 10ops. 


\section{References}

Ayres,T.R., Marstad,N.C. \& Linsky,J.L., 1981, Astrophys. J. 247, 545. Cruddace,R.G. \& Dupree,A.K., 1981, preprint.

Rosner,R., Tucker,W.H. \& Vaiana,G.S., 1978, Astrophys. J. 220, 643. Rucinski,S.M. \& Vilhu,0., 1982, MNRAS, in press.

Vilhu,0. \& Rucinski,S.M., 1982, in prep.

Walter,F., 1982, Astrophys. J. 253, 745. 